\title{
Controlled Artificial Nucleosyntheses and Electron Nuclear Reactor
}

\author{
Alexander Bolonkin
}

\begin{abstract}
By now, about 3000 nuclides are known, which decay and pass into each other. Many of them can be obtained in nuclear reactors. The author offers a method for searching for the desired nuclides and a simple controlled method for obtaining artificial nuclides, such that in the chain of subsequent decays they contain an alpha decay. With alpha decay, a large amount of nuclear energy is generated that can easily be converted into electricity and reactive traction. This method is simple and safe, does not require large, expensive laser, magnetic installations, million temperatures, it can be used in small and medium engines for cars, airplanes, rockets, space vehicles and for unlimited energy on Earth
\end{abstract}

Index Terms- controlled nucleosyntheses, artificial nucleosyntheses, electron nuclear reactor.

\section{INTRODUCTION}

A radionuclide (radioisotope, radioactive nuclide or radioactive isotope) is an atom that has excess nuclear energy, making it unstable. During those processes, the radionuclide is said to undergo radioactive decay. The radioactive decay can produce a stable nuclide or will sometimes produce a new unstable radionuclide which may undergo further decay. Radioactive decay is a random process at the level of single atoms: it is impossible to predict when one particular atom will decay. However, for a collection of atoms of a single element the decay rate, and thus the half-life $\left(T_{1 / 2}\right)$ for that collection can be calculated from their measured decay constants. The range of the half-lives of radioactive atoms have no known limits and span a time range of over 55 orders of magnitude.

Radionuclides occur naturally or are artificially produced in nuclear reactors, cyclotrons, particle accelerators or radionuclide generators. (see list of nuclides [19]). More than 2400 radionuclides have half-lives less than 60 minutes. Most of those are only produced artificially, and have very short half-lives. For comparison, there are about 253 stable nuclides. (In theory, only 146 of them are stable, and the other 107 are believed to decay (alpha decay or beta decay or double beta decay or electron capture or double electron capture)).

All chemical elements can exist as radionuclides. Even the lightest element, hydrogen, has a well-known radionuclide, tritium. Elements heavier than lead, and the elements technetium and promethium, exist only as radionuclides. (In theory, elements heavier than dysprosium exist only as radionuclides, but the half-life for some such elements (e.g. gold and platinum) are too long to found).

On Earth, naturally occurring radionuclides fall into three categories: primordial radionuclides, secondary radionuclides, and cosmogenic radionuclides.
Radionuclides are produced in stellar nucleosynthesis and supernova explosions along with stable nuclides. Secondary radionuclides are radiogenic isotopes derived from the decay of primordial radionuclides. Cosmogenic isotopes, such as carbon-14, are present because they are continually being formed in the atmosphere due to cosmic rays.

Radionuclides are produced as an unavoidable result of nuclear fission and thermonuclear explosions. The process of nuclear fission creates a wide range of fission products, most of which are radionuclides. Further radionuclides can be created from irradiation of the nuclear fuel (creating a range of actinides) and of the surrounding structures, yielding activation products. This complex mixture of radionuclides with different chemistries and radioactivity makes handling nuclear waste and dealing with nuclear fallout particularly problematic.

Synthetic radionuclides are deliberately synthesised using nuclear reactors, particle accelerators or radionuclide generators.

Radionuclides are used in two major ways: either for their radiation alone (irradiation, nuclear batteries) or for the combination of chemical properties and their radiation (tracers, biopharmaceuticals)

\section{A. Theory of radionuclides.}

\section{Radioactive decay.}

Radioactive decay is the process by which an unstable atomic nucleus loses energy (in terms of mass in its rest frame) by emitting radiation, such as an alpha particle, beta particle with neutrino or only a neutrino in the case of electron capture, or a gamma ray or electron in the case of internal conversion. A material containing such unstable nuclei is considered radioactive. Certain highly excited short-lived nuclear states can decay through neutron emission, or more rarely, proton emission. Radioactive decay is a stochastic (i.e. random) process at the level of single atoms. Except for gamma decay or internal conversion from a nuclear excited state, the decay is a nuclear transmutation resulting in a daughter containing a different number of protons or neutrons (or both). When the number of protons changes, an atom of a different chemical element is created.

Alpha decay occurs when the nucleus ejects an alpha particle (helium nucleus). This is the most common process of emitting nucleons, but highly excited nuclei can eject single nucleons, or in the case of cluster decay, specific light nuclei of other elements. Beta decay occurs in two ways: (i) beta-minus decay, when the nucleus emits an electron and an antineutrino in a process that changes a neutron to a proton, or (ii) beta-plus decay, when the nucleus emits a positron and a neutrino in a process that changes a proton to a neutron. 


\section{Types of decay.}

Early researchers found that an electric or magnetic field could split radioactive emissions into three types of beams. The rays were given the names alpha, beta, and gamma, in increasing order of their ability to penetrate matter. Alpha decay is observed only in heavier elements of atomic number 52 (tellurium) and greater, with the exception of beryllium- 8 which decays to two alpha particles. The other two types of decay are produced by all of the elements. Lead, atomic number 82 , is the heaviest element to have any isotopes stable (to the limit of measurement) to radioactive decay. Radioactive decay is seen in all isotopes of all elements of atomic number 83 (bismuth) or greater. Bismuth-209, however, is only very slightly radioactive, with a half-life greater than the age of the universe; radioisotopes with extremely long half-lives are considered effectively stable for practical purposes.

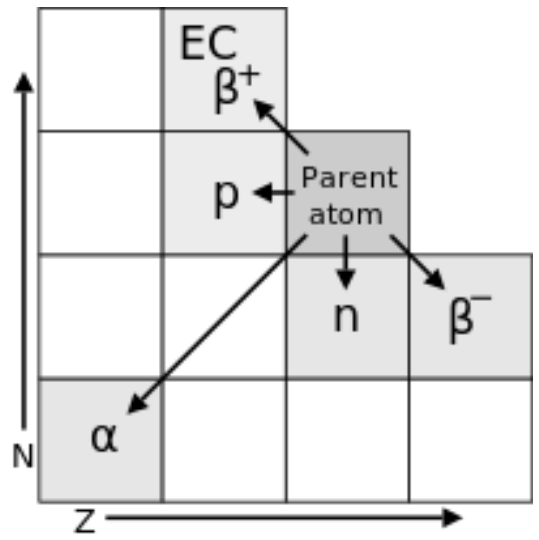

Fig. 1. Transition diagram for decay modes of a radionuclide, with neutron number $N$ and atomic number $Z$ (shown are $\alpha, \beta^{ \pm}$, $\mathrm{p}^{+}$, and $\mathrm{n}^{0}$ emissions, EC denotes electron capture). That is Rule of Soddy.

\section{B. Types of decay related to $N$ and $Z$ numbers.}

\section{Alpha-Decay.}

The Soddy displacement rule for $\alpha$-decay:

$$
{ }_{Z}^{A} \mathrm{X} \rightarrow{ }_{Z-2}^{A-4} \mathrm{Y}+{ }_{2}^{4} \mathrm{He} \text {. }
$$

Here $A$ is atom number, Example (alpha decay of uranium-238 into thorium-234):

$$
{ }_{92}^{238} \mathrm{U} \rightarrow{ }_{90}^{234} \mathrm{Th}+{ }_{2}^{4} \mathrm{He} .
$$

As a result of $\alpha$-decay, the atom is displaced by 2 cells to the beginning of the periodic table (that is, the charge of the Z-nucleus decreases by 2), the mass number of the daughter nucleus decreases by 4 .

\section{Beta $\left(\beta^{-}\right)$-minus decay}

Becquerel proved that $\beta$-rays are an electron flow. Beta decay is a manifestation of weak interaction.

Beta decay (more precisely, beta-minus decay, $\beta$-decay) is a radioactive decay accompanied by the emission of an electron from the nucleus and electron antineutrinos. Beta decay is an intra-nucleated process. Beta-minus decay occurs due to the transformation of one of the $d$-quarks in one of the neutrons of the nucleus into a $u$-quark; In this case, the neutron converts into a proton with the emission of an electron and antineutrinos:

$$
{ }_{0}^{1} \mathrm{n} \rightarrow{ }_{1}^{1} \mathrm{p}+{ }_{-1}^{0} \mathrm{e}+\bar{\nu}_{e} .
$$

Free neutrons also undergo beta decay, turning into a proton, an electron, and an antineutrino (see Beta decay of a neutron).

The Soddy displacement rule for $\beta$ decay: Free neutrons also undergo beta decay, turning into a proton, an electron, and an antineutrino (see Beta decay of a neutron).

The Soddy displacement rule for $\beta$ decay:

$$
{ }_{Z}^{A} \mathrm{X} \rightarrow{ }_{Z+1}^{A} \mathrm{Y}+{ }_{-1}^{0} \mathrm{e}+\bar{\nu}_{e} \text {. }
$$

$\begin{array}{ll}\text { Example: } & \left(\beta^{-} \text {-decay of } \quad \text { Tritium }\right. \\ & { }_{1}^{3} \mathrm{H} \rightarrow{ }_{2}^{3} \mathrm{He}+{ }_{-1}^{0} \mathrm{e}+\bar{\nu}_{e} .\end{array}$ in Helium-3):

After $\beta$-decay, the element shifts by 1 cell to the end of the periodic table (the charge of the nucleus increases by one), while the mass number of the nucleus does not change.

\section{Beta-plus $\left(\beta^{+}\right)$decay and electron capture.}

The Soddy displacement rule for $\beta^{+}$decay and electron capture:

$$
{ }_{Z}^{A} \mathrm{X} \rightarrow{ }_{Z-1}^{A} \mathrm{Y}+e^{+}+\nu_{e}
$$

Example ( $\varepsilon$-electron capture of Beryllium-7 в Lithium-7):

$$
{ }_{4}^{7} \mathrm{Be}+e^{-} \rightarrow{ }_{3}^{7} \mathrm{Li}+\nu_{e} .
$$

After $\beta$-decay, the element shifts by 1 cell to the end of the periodic table (the charge of the nucleus increases by one), while the mass number of the nucleus does not change. Reactions alpha, beta-decay and electron capture produced many energy (up some $\mathrm{MeV}$ ), but in in case beta-decay and electron capture (from lowest electron orbit of atom) the neutrino carries away this energy. Electron bombardment of a nucleus usually converts a proton into a neutron. This means that the number of protons decreases by one, and the number of neutrons increases by one. In the Z-N coordinate system, the element is shifted by one to the left and up, and the mass number A remains unchanged.

$$
{ }_{Z}^{A} \mathrm{X}+e^{-} \rightarrow{ }_{Z-1}^{A} \mathrm{Y}+\nu_{e}
$$

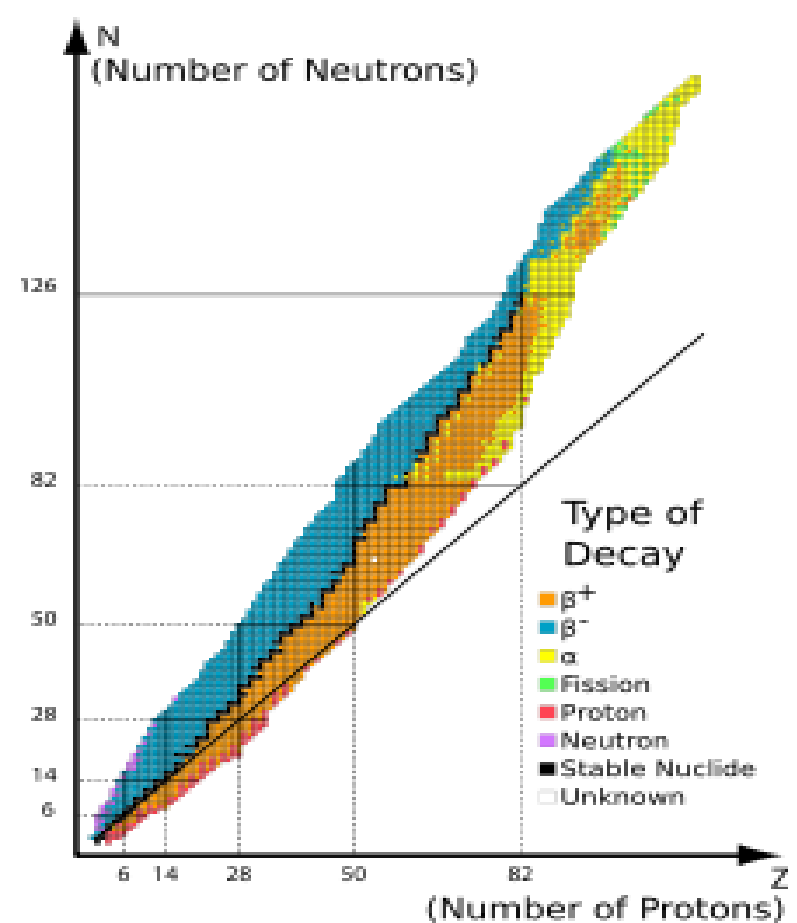

Fig. 2(left). Type of Decay.

https://en.wikipedia.org/wiki/Radioactive_decay\#Decay_mo des_in_table_form 


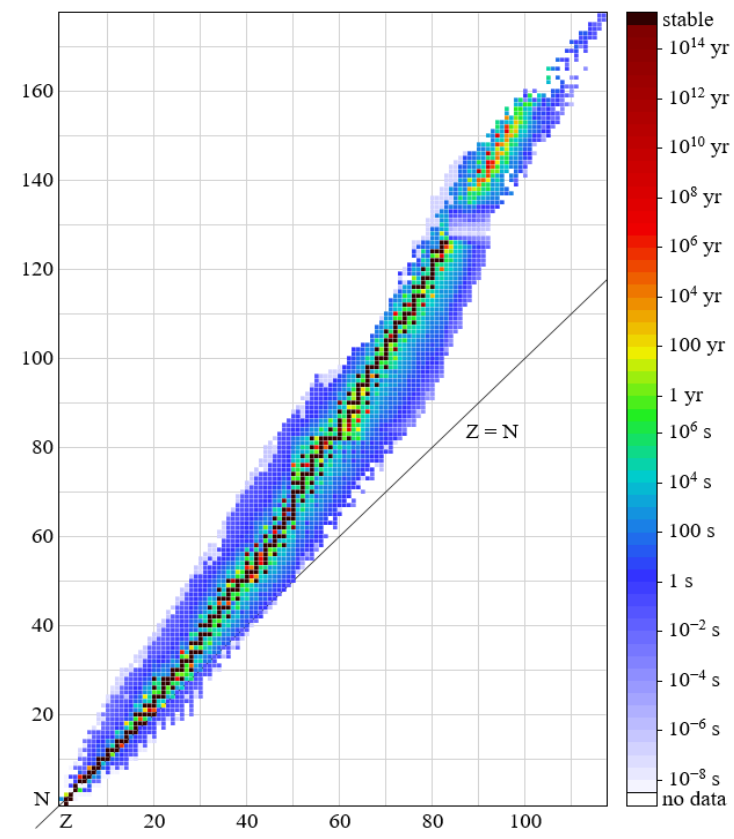

Fig. 3 (right). Half - live.

https://upload.wikimedia.org/wikipedia/commons/8/80/Isoto pes_and_half-life.svg

Computing the total disintegration energy given by the equation:

$$
E=\left(m_{i}-m_{f}-m_{p}\right) c^{2} .
$$

Where $m_{i}$ is the initial mass of the nucleus, $m_{f}$ is the mass of the nucleus after particle emission, and $m_{p}$ is the mass of the emitted particle.

\section{Half-life of radionuclides.}

Half-life (symbol $T_{1 / 2}$ or $\boldsymbol{t}_{1 / 2}$ ) is the time required for a quantity to reduce to half its initial value. There are several formulations of the law, for example, in the form of a differential equation:

$$
\frac{d N}{d t}=-\lambda N
$$

which means that the number of decays $-d N$, which occurred in a short time interval $d t$, is proportional to the number of $N$ atoms in the sample. The solution of this differential equation has the form:

$$
N(t)=N_{0} e^{-\lambda t},
$$

where $N_{0}$ - initial number of atoms in initial time $t=0$.

$$
\begin{aligned}
T_{1 / 2}=\frac{\ln 2}{\lambda} & =\tau \ln 2 \approx 0,693 \tau . \\
\frac{N(t)}{N_{0}} & \approx p(t)=2^{-t / T_{1 / 2}} .
\end{aligned}
$$

where $\tau=1 / \lambda$ is average (mean) life of particles.

\section{Decay chain}

In nuclear science, the decay chain refers to a series of radioactive decays of different radioactive decay products as a sequential series of transformations. It is also known as a "radioactive cascade". Most radioisotopes do not decay directly to a stable state, but rather undergo a series of decays until eventually a stable isotope is reached.

Decay stages are referred to by their relationship to previous or subsequent stages. A parent isotope is one that undergoes decay to form a daughter isotope. One example of this is uranium (atomic number 92) decaying into thorium (atomic number 90). The daughter isotope may be stable or it may decay to form a daughter isotope of its own. The daughter of a daughter isotope is sometimes called a granddaughter isotope.

The time it takes for a single parent atom to decay to an atom of its daughter isotope can vary widely, not only between different parent-daughter pairs, but also randomly between identical pairings of parent and daughter isotopes. The decay of each single atom occurs spontaneously, and the decay of an initial population of identical atoms over time $t$, follows a decaying exponential distribution, $e^{-\lambda t}$, where $\lambda$ is called a decay constant. One of the properties of an isotope is its half-life, the time by which half of an initial number of identical parent radioisotopes have decayed to their daughters, which is inversely related to $\lambda$. Half-lives have been determined in laboratories for many radioisotopes (or radionuclides). These can range from nearly instantaneous to as much as $10^{19}$ years or more.

\section{Example of natural Decay change (Thorium series):}

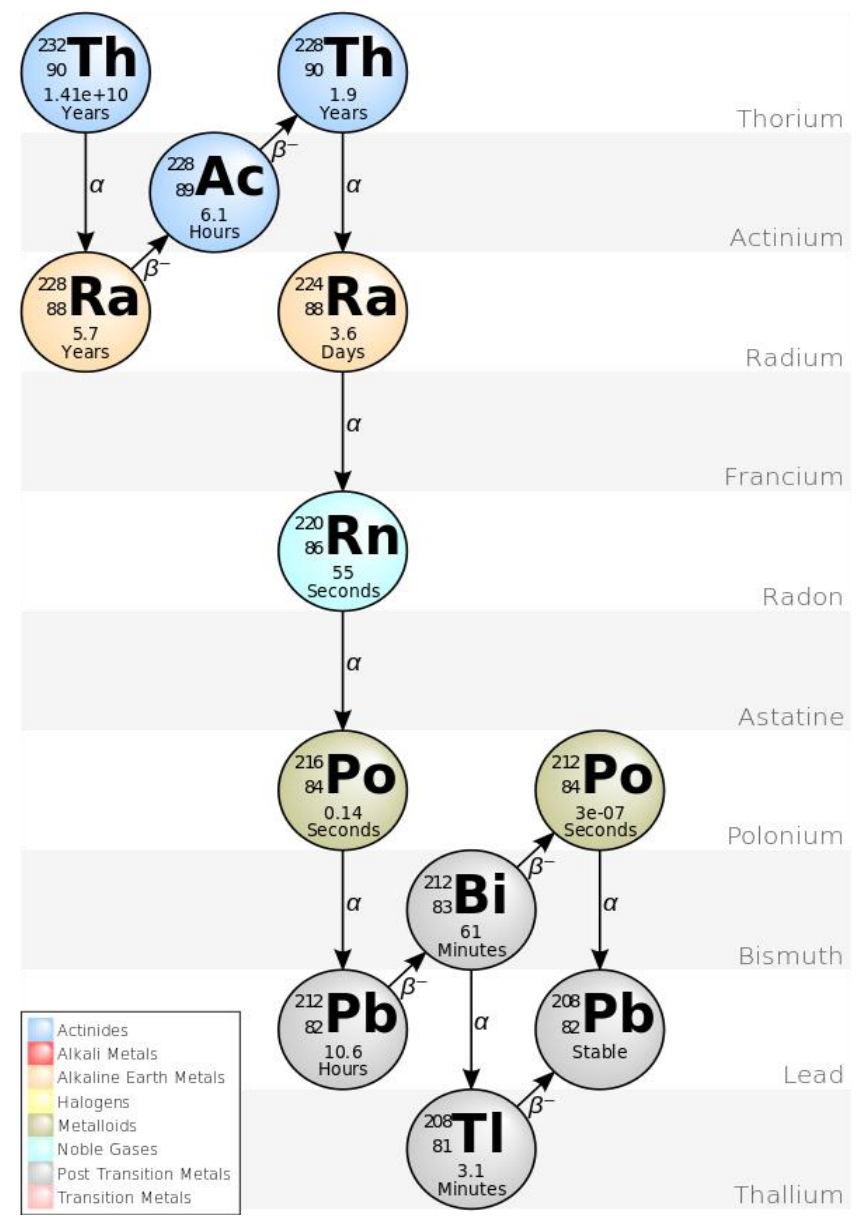

Fig. 4. Thorium series.

The $4 \mathrm{n}$ chain of Th-232 is commonly called the "thorium series" or "thorium cascade". Beginning with naturally occurring thorium-232, this series includes the following elements: actinium, bismuth, lead, polonium, radium, radon and thallium. All are present, at least transiently, in any natural thorium-containing sample, whether metal, compound, or mineral. The series terminates with lead-208 The total energy released from thorium-232 to lead-208, including the energy lost to neutrinos, is $42.6 \mathrm{MeV}$. 
Table \#1, typical isotope-decay.

\begin{tabular}{|c|c|c|c|c|c|c|}
\hline nuclide & $\begin{array}{l}\text { historic } \\
\text { name } \\
\text { (short) }\end{array}$ & $\begin{array}{l}\text { historic } \\
\text { (long) }\end{array}$ & $\begin{array}{l}\text { decay } \\
\text { mode }\end{array}$ & $\begin{array}{l}\text { half-life } \\
(a=\text { year })\end{array}$ & $\begin{array}{l}\text { energy } \\
\text { released, } \\
\mathrm{MeV}\end{array}$ & $\begin{array}{l}\text { product } \\
\text { of decay }\end{array}$ \\
\hline${ }^{252} \mathrm{Cf}$ & & & $\alpha$ & $2.645 \mathrm{a}$ & 6.1181 & ${ }^{248} \mathrm{Cm}$ \\
\hline${ }^{248} \mathrm{Cm}$ & & & $\alpha$ & $3.4 \times 10^{5} \mathrm{a}$ & 5.162 & ${ }^{244} \mathrm{Pu}$ \\
\hline${ }^{244} \mathrm{Pu}$ & & & $\alpha$ & $8 \times 10^{7}$ a & 4.589 & ${ }^{240} \mathrm{U}$ \\
\hline${ }^{240} \mathrm{U}$ & & & $\beta^{-}$ & $14.1 \mathrm{~h}$ & .39 & ${ }^{240} \mathrm{~Np}$ \\
\hline${ }^{240} \mathrm{~Np}$ & & & $\beta^{-}$ & $1.032 \mathrm{~h}$ & 2.2 & ${ }^{240} \mathrm{Pu}$ \\
\hline${ }^{240} \mathrm{Pu}$ & & & $\alpha$ & $6561 \mathrm{a}$ & 5.1683 & ${ }^{236} \mathrm{U}$ \\
\hline${ }^{236} \mathrm{U}$ & & Thoruranium & $\alpha$ & $2.3 \times 10^{7} \mathrm{a}$ & 4.494 & ${ }^{232} \mathrm{Th}$ \\
\hline${ }^{232} \mathrm{Th}$ & Th & Thorium & $\alpha$ & $1.405 \times 10^{10} \mathrm{a}$ & 4.081 & ${ }^{228} \mathrm{Ra}$ \\
\hline${ }^{228} \mathrm{Ra}$ & $\mathrm{MsTh}_{1}$ & Mesothorium 1 & $\beta^{-}$ & $5.75 \mathrm{a}$ & 0.046 & ${ }^{228} \mathrm{Ac}$ \\
\hline${ }^{228} \mathrm{Ac}$ & $\mathrm{MsTh}_{2}$ & Mesothorium 2 & $\beta^{-}$ & $6.25 \mathrm{~h}$ & 2.124 & ${ }^{228} \mathrm{Th}$ \\
\hline${ }^{228} \mathrm{Th}$ & RdTh & Radiothorium & $\alpha$ & $1.9116 \mathrm{a}$ & 5.520 & ${ }^{224} \mathrm{Ra}$ \\
\hline${ }^{224} \mathrm{Ra}$ & ThX & Thorium X & $\alpha$ & $3.6319 \mathrm{~d}$ & 5.789 & ${ }^{220} \mathrm{Rn}$ \\
\hline${ }^{220} \mathrm{Rn}$ & $\mathrm{Tn}$ & $\begin{array}{l}\text { Thoron, } \\
\text { Thorium Emanation }\end{array}$ & $\alpha$ & $55.6 \mathrm{~s}$ & 6.404 & ${ }^{216} \mathrm{Po}$ \\
\hline${ }^{216} \mathrm{Po}$ & ThA & Thorium A & $\alpha$ & $0.145 \mathrm{~s}$ & 6.906 & ${ }^{212} \mathrm{~Pb}$ \\
\hline${ }^{212} \mathrm{~Pb}$ & ThB & Thorium B & $\beta^{-}$ & $10.64 \mathrm{~h}$ & 0.570 & ${ }^{212} \mathrm{Bi}$ \\
\hline${ }^{212} \mathrm{Bi}$ & ThC & Thorium C & $\begin{array}{l}\beta^{-} \\
64.06 \% \\
\alpha \\
35.94 \%\end{array}$ & $60.55 \mathrm{~min}$ & $\begin{array}{l}2.252 \\
6.208\end{array}$ & $\begin{array}{l}{ }^{212} \mathrm{Po} \\
{ }^{208} \mathrm{Tl}\end{array}$ \\
\hline${ }^{212} \mathrm{Po}$ & $\mathrm{ThC}^{\prime}$ & Thorium $\mathrm{C}^{\prime}$ & $\alpha$ & $299 \mathrm{~ns}$ & 8.955 & ${ }^{208} \mathrm{~Pb}$ \\
\hline${ }^{208} \mathrm{Tl}$ & $\mathrm{ThC}^{\prime \prime}$ & Thorium C" & $\beta^{-}$ & $3.053 \mathrm{~min}$ & 4.999 & ${ }^{208} \mathrm{~Pb}$ \\
\hline${ }^{208} \mathrm{~Pb}$ & $\mathrm{ThD}$ & Thorium D & stable & & & \\
\hline
\end{tabular}

Base idea. Control Artificial Nucleosyntheses.

The modern theory of the universe says: after the Big Bang and the Great Synthesis, thousands of unstable isotopes with different periods of existence arose. Over the billions of years of the existence of the universe, their time of life has come to an end and almost two hundred isotopes, whose lifetime is longer than the time of existence of the universe (or a given planet), have practically remained. Some of the short-lived isotopes are formed in the hot subsurface of the stars or by the action of cosmic rays. Using nuclear reactors, people have learned to create artificial radioactive isotopes, which are known to date about 3000 . They can turn into each other and give off giant energy. Unfortunately, the lifetime (decay) of these isotopes is strictly prescribed and it is practically impossible to regulate it with modern technologies. This greatly limits their practical application. In addition, many of the artificial isotopes are expensive and there is a very short time. The author's task is to circumvent these obstacles. But not in order, as in ancient alchemy, to turn a certain matter into gold, but in order to solve the main problem of our time to have an abundance of cheap energy. Currently, the government and scientists are trying to solve the problem of energy using nuclear power by the Fission or Fusion methods. The Fission method has been implemented, dozens of powerful uranium nuclear power plants have been constructed, but their energy is not cheaper than fuel cells on natural fuel. In addition, they were very dangerous in the event of an accident. Over the Fusion method, scientists have been working for more than 60 years in all industrialized countries. Governments have spent hundreds of billions of US dollars, but there are no results and will not be recognized by scientists in the next 15-20 years.

The essence of the author's idea: in the Fusion method, we connect two negatively charged nuclei, naming $\mathrm{T}+\mathrm{D}$ (tritium and deuterium) to obtain energy. This task is very complicated. Both atoms are positively charged and repelled from each other. We heat the mixture to tens of millions of degrees and compress it to hundreds of thousands of atmospheres to make the reaction happen. In the proposed method, negatively charged electrons bombard a positively charged nucleus and attract each other, acquiring an additional velocity (kinetic energy). In the event of an electron falling into the nucleus, we get a new isotope shifted in the isotope table to the cell to the left and up. In many cases, nuclear energy is released. But for us it is useless, for it immediately takes away from it. The neutrino has almost zero mass and therefore takes almost all energy. Neutrinos have a giant penetrating power and its energy cannot be used by modern technology. That's why scientists did not pay attention to the study of such a reaction. But a new isotope can have alpha-decay or become the beginning of a chain that has alpha decay in its composition (see Fig. 4). We can use alpha decay for direct conversion to electrical energy and reactive traction with a very high specific impulse. The ability (probability) to get into the nucleus (to cause the desired reaction) is characterized by the so-called cross section of the reaction. The author was unable to find experimental data on the cross section of electron-nuclides. The scientists of NIST USA could not help him either. They only indicated that this cross section cannot be less than the target area of the target nucleus. The fact that the reaction of the electron-nucleus of the nuclide exists no doubt, for the natural reactions of the electron-nucleus exist. Moreover, this is the only reaction of natural decay in nature, the probability of which depends on the temperature and pressure. This influence is small (about 1\%), but the electron energy in the nearest orbit is small (about $10 \mathrm{eV}$ ). We can easily disperse the attacking electrons to tens and hundreds of thousands of $\mathrm{eV}$. By regulating the number and energy of electrons, we can regulate the number of new nuclides that are obtained, and hence the amount of energy received.

But there are some technical limitations: we must start with an inexpensive nuclide that has half-time at least a month or more. Otherwise, the initial fuel will quickly deteriorate. Isotopes in the chain and obtained before the first desired isotope (alpha-decay or neutron) should have a small half-time (less $1 \mathrm{sec}$.). Otherwise, the engine (rocket) will react slowly to control, which in many cases is unacceptable. Note that we adjust not the probability of decay of the isotope $\lambda$, but the amount of the initial isotope participating in the reaction, i.e. coefficient $N_{0}$ in the formula (10).

The method of searching for the desired nuclides.

The number of known and studied (in the sense of decay) isotopes to date is about 3,000. Many of them can decay simultaneously through many channels. Each isotope channel has its own probability percentage, its decay time and its decay energy. Therefore, the search for the desired decay chain is a laborious task (graph theory). The solution to this problem is possible on a computer (super-computer). Branches leading to a dead end (large half-time) should be cut off. The search ends when we get the right amount of energy (alpha process) or the desired element (or isotope with half-time satisfying us). The selected nuclear fuel chain must satisfy three conditions:

1) Fuel should be inexpensive.2) The initial element of the chain must have sufficient disintegration time (for vehicles - a storage period of at least some days). 3) In the final element of the chain should quickly alpha decay (less than one second for vehicles). 


\section{International Journal of Engineering and Technical Research (IJETR) \\ ISSN: 2321-0869 (O) 2454-4698 (P) Volume-8, Issue-12, December 2018}

To find the right fuel, author suggests two methods: 1) Start from a sufficiently stable element and bombard it with electrons to move down the A-constant chain in the hope that it will contain an element with a short alpha half-life. 2) Start from an element with a short alpha decay period and bombard it with electrons to move up the A-constant chain in the hope that it will have a fairly stable element. If you have a source of slow (thermal) neutrons, then you can explore the side branches of this chain. You examine the series:

$$
{ }^{\mathrm{A}} \mathrm{Y}_{\mathrm{Z}} \leftarrow{ }^{\mathrm{A}} \mathrm{Y}_{\mathrm{Z}+1}+\mathrm{e} \leftarrow{ }^{\mathrm{A}} \mathrm{Y}_{\mathrm{Z}+2}+\mathrm{e} \leftarrow \ldots
$$

and select in it the starting element (initial nuclear fuel, that has need half-time which is the satisfaction of you). Here $e$ means the bombing this element by electron. The reader can find the initial data for selection, in particular, in [18]. Using the filter [18] you can get the list of nuclides ${ }^{\mathrm{A}} \mathrm{Y}_{\mathrm{Z}}$ having $\alpha$-decay mode and the half-time less given value. You examine the series (14) and select in it the starting element (initial nuclear fuel, that has need half-time which is the satisfaction of you). Here $e$ means the bombing this element by electron. If you are selecting fuel for a transport engine, where the response time is important, then half-time of the isotopes between the initial and final isotope should in sum have a time of less than 1 second. In the first method: you start with a stable or long-term isotope and by bombarding electrons, neutrons, it is possible to obtain an alpha-isotope with the right time.

\section{ADVANTAGES OF THE PROPOSED METHOD.}

The proposed method for obtaining nuclear energy (alpha decay) has huge advantages over the Fission and Fusion methods currently used:

1. It uses scanty amounts of nuclear fuel (a fraction of a milligram) and can be used on small and medium-sized engines (cars, airplanes, missiles) [1-3]. The engine can be

switched on and off at any time. 2. It is safe. Destruction of the engine will not lead to contamination of the terrain.

3. It does not need huge and expensive reactors. The technology of production of the

focused electron beam is well worked out (we recall the old volume TVs).

4. Compared to the Fusion method, the proposed method does not require giant laser or magnetic installations, in quasi-zero and millionth temperatures.

5. In principle, it allows you to immediately receive electrical energy and, in most cases, does not produce neutrons that make the materials radioactive (the beta radiation is delayed even by a sheet of paper and air). 6. In principle, it allows to obtain artificial elements.

\section{DISCUSSING}

Mankind has long been dreaming of mastering unlimited energy. But such an opportunity arose when scientists discovered huge reserves of nuclear energy. Two methods of obtaining it are possible: the method of decay of large complex nuclei into simpler ones and the method of merging simple nuclei into more complex ones.

Scientists quickly developed and mastered the technology of decay (Fission) methods. But it turned out that it is complex, expensive, and most importantly very dangerous. Technology requires a large mass of radioactive matter, which in the event of an accident infects large areas. The second method - the fusion of light nuclei, is being developed for more than 60 years. Governments spent tens of billions of dollars on it, but there is no result and it is not expected soon. In addition, estimates show that the cost of energy received by the current Fusion method will be much greater than the current energy prices. The author proposes a new method for obtaining nuclear energy. For a long time ( 100 years) it is known that isotopes decay themselves with the release of energy. But we cannot in any way influence this natural disintegration. In addition, only recently with the advent of nuclear reactors, it became possible to obtain isotopes.

The author proposes to obtain isotopes by bombarding nuclei with electrons. The method of bombarding nuclei with nuclei or neutrons for the purpose of obtaining energy is known and developed long ago. But electron bombardment leads in most cases to beta decay, which does not give practical energy, and apparently nobody studied the bombardment of the nucleus by electrons. Apparently the choice of the initial nucleus can be obtained immediately by the nucleus subject to alpha decay or a chain of decays, which in the end contains a rapid alpha decay.

Essentially, another proposal is the management of disintegration. The author does not encroach on the law of independence of the rate of decay from the temperature and pressure of the environment (although in the case of beta decay such a dependence exists). Changing the speed and intensity of the electron beam, it changes the number (concentration) of the initial and daughter nuclei in the isotope, and thus the amount of energy released. Note that the implementation of the author's proposals is possible only in a simple reactor proposed by the author in [1-8], using the proposed isotope fuels. Since the proposed method does not use high nuclear fusion temperatures, the proposed method can be attributed to the so-called "cold nuclear reactor"[8] (room temperature).

\section{RÉFÉRENCES}

[1] Bolonkin A.A., Inertial Impulse Beam Hole Thermonuclear AB Reactor www.IntellectualArchive.com, \#1972, 87 18. http://GSJournal.net http://viXra.org/abs/1808.0089

[2] Bolonkin A.A., Provisional (patent) application \# 62729486. Inertial Impulse Electric Thermonuclear Reactor and Method of It. https://archive.org/details/

ProvisionalPatentInertialElectricReactor81218, www.IntellectualArchive.com, \#1977.

[3] Bolonkin A.A., Small, Non-Expensive Electric Impulse Thermonuclear Reactor with colliding jets. 711 16, 1119 16, LULU 2017, 144 ps, http://viXra.org/abs/1611.0276,

//archive.org/download/ArticleThermonuclearReactorOfCollisingJets1 0416,

[4] Bolonkin A.A. , Electric Cumulative Thermonuclear Reactors. 71716 http://vixra.org/abs/1610.0208

https://archive.org/details/abolonkin_gmail_201610,

[5] Bolonkin A.A., "Inexpensive Mini Thermonuclear Reactor". International Journal of Advanced Engineering Applications, Vol.1, Iss.6, pp.62-77 (2012). http://viXra.org/abs/1305.0046 http://archive.org/details/InexpensiveMiniThermonuclearReactor

[6] Bolonkin A.A. , Cumulative Thermonuclear AB-Reactor. Vixra 7/ $8 / 2015$

https://archive.org/details/ArticleCumulativeReactorFinalAfterCathAn dOlga7716

http://viXra.org/abs/1507.0053 . 
[7] Bolonkin A.A., Ultra-Cold Thermonuclear Synthesis: Criterion of Cold Fusion. 718 2015.http://viXra.org/abs/1507.0158, GSJornal: http://gsjournal.net/Science-

Journals/\%7B \$cat_name\%7D/View/6140 .

[8] Bolonkin A.A., Cumulative and Impulse Mini Thermonuclear Reactors. 33016

https://archive.org/download/ImpulseMiniThermonuclearReactors http://viXra.org/abs/1605.0309,

[9] Bolonkin, A.A., "Non Rocket Space Launch and Flight". Elsevier, 2005. 488 pgs. ISBN-13:978-0-08044-731-5, ISBN-10: 0-080-44731-7 . http://vixra.org/abs/1504.0011 v4,

[10] Bolonkin, A.A., "New Concepts, Ideas, Innovations in Aerospace, Technology and the Human Sciences", NOVA, 2006, 510 pgs. ISBN-13: 978-1-60021-787-6. http://viXra.org/abs/1309.0193.

[11] Bolonkin, A.A., Femtotechnologies and Revolutionary Projects. Lambert, USA, 2011. $538 \quad$ p. $16 \quad$ Mb. ISBN:978-3-8473-0839-0.http://viXra.org/abs/1309.0191,

[12] Bolonkin, A.A., Innovations and New Technologies (v2). Lulu, 2014. 465 pgs. $\quad 10.5 \quad \mathrm{Mb}, \quad$ ISBN: 978-1-312-62280-7.

https://archive.org/details/Book5InnovationsAndNewTechnologiesv21 02014/

[13] Bolonkin, A.A., Stability and Production Super-Strong AB Matter. International Journal of Advanced Engineering Applications. 3-1-3,
February
2014 ,
pp. $18-33$

http://fragrancejournals.com/wp-content/uploads/2013/03/IJAEA-3-1-

3.pdf The General Science Journal, November, 2013, \#5244.

[14] Bolonkin, A.A., Converting of Any Matter to Nuclear Energy by AB-Generator. American Journal of Engineering and Applied Science, Vol. 2, \#4, 2009, pp.683-693. http://viXra.org/abs/1309.0200.

[15] Kikoin I.K., Tables of. Physical Values, Moscow, Atomizdat, 1975 (Russian).

[16] Koshkin N.I., Shirkevich M.G., Handbook of elementary physics, Moscow, Nauka, 1982 (Russian).

[17] AIP, Physics Desk Reference, AIP PRESS. Third Edition.

[18] Data of nuclides: https://www-nds.iaea.org/relnsd/vcharthtml/VChartHTML.html

[19] Wikipedia: https://en.wikipedia.org/wiki/List_of_nuclides 24 September 2018 